\title{
Article \\ The Drosophila miR-959-962 Cluster Members Repress Toll Signaling to Regulate Antibacterial Defense during Bacterial Infection
}

\author{
Ruimin Li, Xiaolong Yao, Hongjian Zhou, Ping Jin * and Fei Ma *

\begin{abstract}
Laboratory for Comparative Genomics and Bioinformatics \& Jiangsu Key Laboratory for Biodiversity and Biotechnology, College of Life Science, Nanjing Normal University, Nanjing 210046, China; 161201003@njnu.edu.cn (R.L.); 181202093@njnu.edu.cn (X.Y.); 181201007@njnu.edu.cn (H.Z.)
\end{abstract} \\ * Correspondence: jinping@njnu.edu.cn (P.J.); mafei01@tsinghua.org.cn (F.M.); Tel.: +86-25-85891852 (P.J.); \\ +86-25-85891852 (F.M.)
}

check for

updates

Citation: Li, R.; Yao, X.; Zhou, H.; Jin, P.; Ma, F. The Drosophila miR-959-962

Cluster Members Repress Toll

Signaling to Regulate Antibacterial Defense during Bacterial Infection. Int. J. Mol. Sci. 2021, 22, 886 https://doi.org/10.3390/ijms 22020886

Received: 11 December 2020

Accepted: 14 January 2021

Published: 17 January 2021

Publisher's Note: MDPI stays neutral with regard to jurisdictional clai$\mathrm{ms}$ in published maps and institutional affiliations.

Copyright: (C) 2021 by the authors. Licensee MDPI, Basel, Switzerland. This article is an open access article distributed under the terms and conditions of the Creative Commons Attribution (CC BY) license (https:// creativecommons.org/licenses/by/ $4.0 /)$.

\begin{abstract}
MicroRNAs (miRNAs) are a class of $\sim 22 \mathrm{nt}$ non-coding RNA molecules in metazoans capable of down-regulating target gene expression by binding to the complementary sites in the mRNA transcripts. Many individual miRNAs are implicated in a broad range of biological pathways, but functional characterization of miRNA clusters in concert is limited. Here, we report that miR-959-962 cluster (miR-959/960/961/962) can weaken Drosophila immune response to bacterial infection evidenced by the reduced expression of antimicrobial peptide Drosomycin (Drs) and short survival within $24 \mathrm{~h}$ upon infection. Each of the four miRNA members is confirmed to contribute to the reduced Drs expression and survival rate of Drosophila. Mechanically, RT-qPCR and Dual-luciferase reporter assay verify that tube and dorsal ( $d l)$ mRNAs, key components of Toll pathway, can simultaneously be targeted by miR-959 and miR-960, miR-961, and miR-962, respectively. Furthermore, miR-962 can even directly target to the $3^{\prime}$ untranslated region (UTR) of Toll. In addition, the dynamic expression pattern analysis in wild-type flies reveals that four miRNA members play important functions in Drosophila immune homeostasis restoration at the late stage of Micrococcus luteus (M. luteus) infection. Taken together, our results identify four miRNA members from miR-959-962 cluster as novel suppressors of Toll signaling and enrich the repertoire of immune-modulating miRNA in Drosophila.
\end{abstract}

Keywords: miR-959-962 cluster; Toll pathway; Toll; tube; dl; Drosophila melanogaster

\section{Introduction}

For the host, an appropriate immune response is essential to resist various pathogenic microorganisms and maintain the immune homeostasis. However, uncontrolled immune response would be detrimental to the host, eventually leading to the acute and chronic inflammatory disorders [1]. Determined by the speed and the specificity of the reaction, the innate and the adaptive immunities are vital for animal's survival [2]. While invertebrates, such as Drosophila melanogaster, rely exclusively on innate immunity, as the first-line defense against microbial invaders $[3,4]$. The response of the flies to bacterial and fungal infections involves two main evolutionary conserved signaling pathways, Toll and immune deficiency (Imd) [5,6] which have been well-established. Upon systemic Gram-positive bacterial or fungal infection via septic injury, the Toll pathway is triggered, which involves extracellular proteolytic cascades activated by secreted recognition molecules (PGRP-SA, PGRP-SD, GNBP1, and GNBP3) [7-11]. Next, the transmembrane receptor Toll is activated and dimerized by the mature proteolytic product Spätzle [12-15], which subsequently causes the recruitment of three intracellular Death domain-containing proteins, MyD88, Tube, and Pelle [16-18]. Then the IкB homologue Cactus is phosphorylated and degraded by the proteasome, leading to the release of members of the nuclear factor NF- $\kappa$ B family (Dif or 
Dorsal) to translocate to the nucleus [19-21], and activation of genes encoding potent antifungal and anti-bacterial peptides, such as Drosomycin [7,22,23]. In addition, in response to Gram-negative bacterial infection, the Imd pathway is activated, eventually resulting that another Drosophila NF- $\mathrm{kB}$ family member Relish moves from the cytoplasm to the nucleus, and the expression of antimicrobial peptide (AMP) genes, such as Diptericin [22,24] Therefore, the Toll and Imd immune pathways work together and constitute a robust defense system that protects Drosophila from invading pathogens [5].

The inactivation or overactivation of the immune response could lead to the damage of the normal tissue. Therefore, the activation and termination of the Toll pathway require the cooperation of various molecules at multiple stages to establish a complete immune regulatory system. At present, kinds of modulators have been identified to be involved in Toll pathway regulation. For example, five serine proteases (ModSP, Grass, Spirit, Spheroide, and Sphinx1/2), are considered as essential for host resistance to fungal and Gram-positive infection, which play a vital role in the extracellular proteolytic cascades linking the signaling recognition proteins and Spz $[25,26]$. In addition, a highly conserved protein Pellino, has shown to act as a positive regulator of Toll signaling by interacting with activated Pelle kinase [27]. Furthermore, in a genome-wide RNAi screens in S2 cells, G Protein-coupled receptor kinase 2 (Gprk2) was identified as a regulator of the Toll pathway [28], and the transcription factor DEAF-1 is confirmed to be required to induce Toll pathway target genes at or downstream of Dif/Dorsal [29]. Lastly, a feedback inhibitor is WntD, which reduces Toll activity by preventing translocation of Dorsal to the nucleus [30].

In addition to the above-mentioned protein regulatory factors, recently, growing evidences have exhibited that miRNA controls are a critical regulator in the immune response process via Toll pathway [31]. miRNAs could fine tune gene expression in diverse cellular and biological processes, through perfect or imperfect base-pairing to the $3^{\prime}$ UTR of the target mRNAs, resulting in cleavage or degradation of the target mRNAs or suppression of their translation [32,33]. For example, the transmembrane receptor Toll protein is a crucial factor connecting extracellular and intracellular signals, and it has been reported that miR-8 [34] and miR-958 [35] can target the $3^{\prime}$ UTR of its mRNA to negatively modulate the Toll pathway. Moreover, the nuclear translocation of the transcription factor Dif or Dorsal and its activation of AMP expression are an indispensable step of the Toll pathway response. miR-958 [35] and miR-317 [36] have been identified the direct binding with the $3^{\prime}$ UTR of Dif-Ra/b/d and Dif-Rc transcripts, respectively, while miR-8 targets to the Dorsal mRNA [34]. Last but not least, miR-310-313 family and miR-964 could directly target to the AMP gene Drosomycin to inhibit its expression [37,38]. Although several regulators involved in Drosophila Toll-mediated immune response have been identified, the restoration mechanism of Drosophila immune homeostasis is still largely unknown and needs for further research.

Especially, in our previous work, we found that the high-expression of four members of this miR-959-962 cluster could significantly down-regulate Drs expression via RNA-seq analysis and multiple genetic screening works [37]. Whether Drosophila miR-959-962 cluster members can synergistically repress Toll signaling to stop an overactive immune response, which is still not clear. In this study, we further investigated the regulatory mechanism of miR-959-962 cluster in the Drosophila immune response to bacterial infection. Each individual miRNA from the miR-959-962 cluster could reduce the survival rate of flies via inhibiting the expression of AMP Drs. Bioinformatics prediction and in vitro/in vivo experiments verified that four miRNA members (miR-959, miR-960, miR-961, and miR-962) could negatively regulated the Toll pathway in combination via directly targeting the $3^{\prime}$ UTR of tube, $d l$, or Toll mRNA. In addition, the dynamic expression pattern analysis demonstrated that four miRNA members were up-regulated at the late stage of M. luteus infection, revealing their important functions in the immune homeostasis restoration of Drosophila. Overall, our results have clarified that four miRNA members from miR-959-962 cluster are novel negative regulators in Drosophila Toll-mediated immune homeostasis restoration and their aberrant expression seriously influence Drosophila antibacterial defenses. 


\section{Results}

2.1. The miR-959-962 Cluster Could Negatively Regulate Drosophila Toll-Related Immune Response

In order to assess the role of miR-959-962 cluster in Drosophila immune response, we first observed whether miR-959-962 cluster dysregulation would affect the resistance of Drosophila in response to lethal Gram-positive bacterial infection, Enterococcus faecalis (E. faecalis). As shown in Figure 1, the flies transiently overexpressed miR-959-962 cluster $\left(\right.$ Gal80 $^{t s}$; Tub > miR-959-962) under a temperature sensitive control and had a lower survival rate than the control flies (Gal80 ${ }^{t s}$; Tub-Gal4/+) (Figure 1A). While the survival rate of the flies with miR-959-962 cluster knockout (miR-959-962 KO) was obviously increased, compared with the wild-type flies $\left(w^{1118}\right)$ (Figure 1B). We also confirmed the exact overexpression (Figure S1) and knockout (Figure S2) of each miRNA in the corresponding flies. These results suggest that the miR-959-962 cluster could weaken Drosophila antibacterial defense, implying a role for miR-959-962 cluster members in the negative regulation of Toll signaling.

A

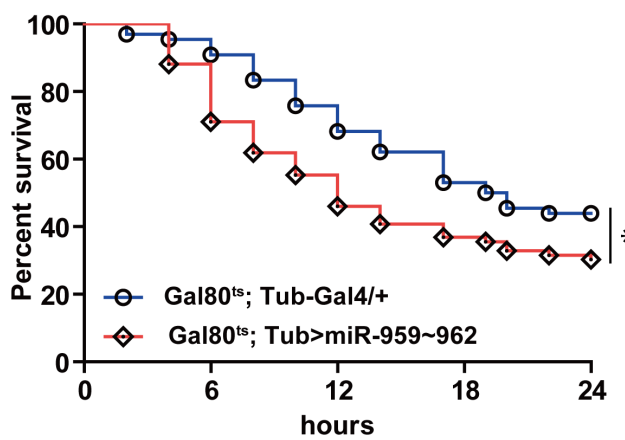

C

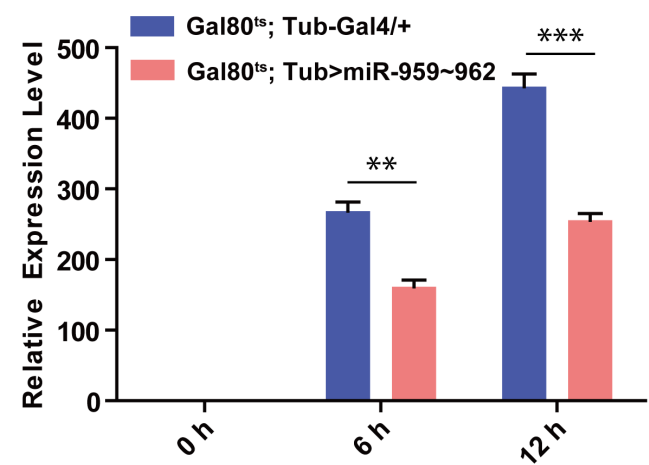

B

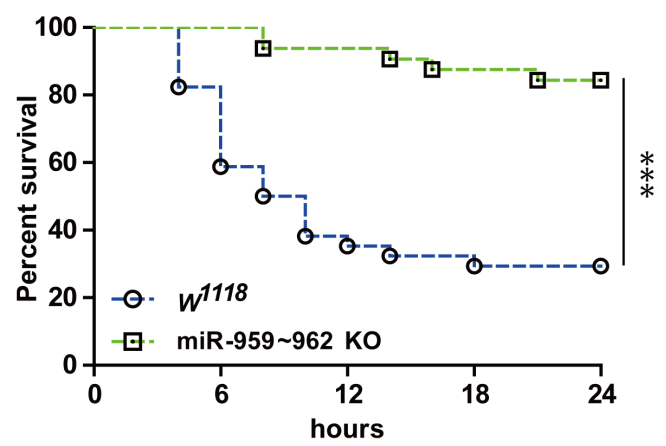

E

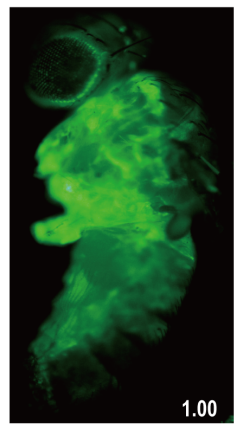

Drs-GFP; Gal80"s; Tub-Gal4/+

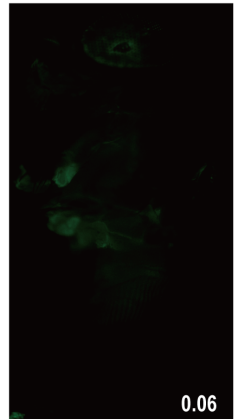

Drs-GFP; Gal80ts; Tub>UAS-miR-959 962

Figure 1. Cont. 
D

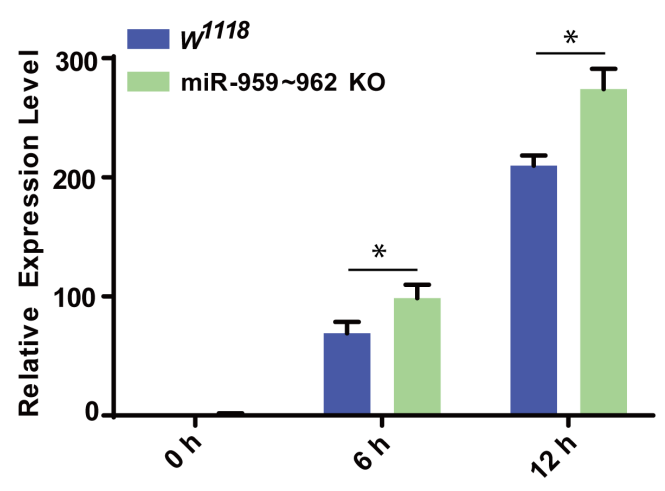

F

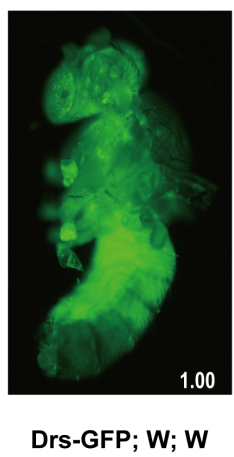

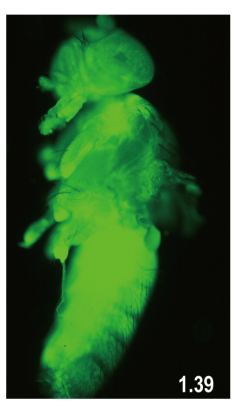

Drs-GFP; miR-959 962 KO;

Figure 1. The miR-959-962 cluster negatively regulates Drosophila Toll-related immune response. (A) The changes of the survival rate were observed both in miR-959-962 cluster high-expressing flies (Gal80 ${ }^{\text {ts }}$ Tub > UAS-miR-959-962) and the control (Gal80 ${ }^{\text {ts }}$; Tub-Gal4/+) flies with E. faecalis infection. (B) The changes of the survival rate were observed both in miR-959-962 knock-out flies (miR-959-962 KO) and the control $\left(w^{1118}\right)$ flies upon E. faecalis infection. The expression levels of AMP Drs were examined in miR-959-962 cluster high-expressing flies (C) and miR-959-962 knock-out flies (D) at 0, 6 and $12 \mathrm{~h}$ upon M. luteus infection. (E) The green-fluorescent in miR-959-962 cluster high-expressing flies (Drs-GFP; Gal80 ${ }^{t s}$; Tub > UAS-miR-959-962, right) and the controls (Drs-GFP; Gal80 ${ }^{t s}$; Tub-Gal4/+, left) carrying with Drs-GFP reporter gene were observed under fluorescent microscope following infection with M. luteus. (F) The green-fluorescent in miR-959-962 cluster knock-out flies (Drs-GFP; miR-959-962 KO; $w$, right) and the controls (Drs-GFP; $w ; w$, left) carrying with Drs-GFP reporter gene were observed under fluorescent microscope following infection with $M$. luteus. The levels of GFP were quantified using Image J software with the default parameters and their relative level values were marked in the bottom right corner of the image. $\left({ }^{*} p<0.05 ;{ }^{* *} p<0.01\right.$; $\left.{ }^{* *} p<0.001\right)$.

To further confirm the effect of miR-959-962 cluster on the Toll pathway, we monitored the mRNA expression level of the AMP Drs, as the readout of Toll pathway activation, in the flies with miR-959-962 cluster overexpression and knockout before and after M. luteus infection. A significant reduction of Drs level was observed in miR-959-962 cluster overexpressing flies at $6 \mathrm{~h}$ and $12 \mathrm{~h}$ under bacterial challenge, compared with the corresponding control groups (Figure 1C). On the contrary, a higher expression level of Drs was detected in miR-959-962 KO flies than in wild-type controls (Figure 1D). Likewise, taking advantage of a Drosomycin-green fluorescent protein (GFP) reporter fly strain (Drs-GFP), we also observed that overexpression of miR-959-962 cluster inhibited the expression of Drs in live flies (94\%) (Figure 1E), while the knock-out of miR-959-962 cluster increased the expression of Drs in live flies (39\%) (Figure 1F). Our results suggest that four members of the miR-959-962 cluster may synergistically downregulate Toll signaling response to prevent overactivation of immune response and maintain Drosophila innate immune homeostasis.

\subsection{Each Member from miR-959-962 Cluster Plays a Negative Regulatory Role in Drosophila Toll Pathway}

To further explore the role of each miRNA individual from miR-959-962 cluster in Drosophila Toll pathway, transgenic lines high-expressing miR-959, miR-960, miR-961 or miR-962 separately (confirmed using RT-qPCR in Figure S3) were infected with M. luteus. The expression levels of Drs at $6 \mathrm{~h}$ and $12 \mathrm{~h}$ post-infection were also detected by RT-qPCR. Our result revealed that the Drs mRNA levels in the flies with miR-959 high-expression $\left(\right.$ Gal80 $^{t s} ;$ Tub > miR-959) (Figure 2A), miR-960 high-expression $\left(\right.$ Gal80 ${ }^{\text {ts }}$; Tub > miR-960) (Figure 2B), miR-961 high-expression (Gal80 ${ }^{t s}$; Tub > miR-961) (Figure 2C), or miR-962 high-expression (Gal80 ${ }^{t s}$; Tub > miR-962) (Figure 2D) were significantly lower than that in the control flies (Gal80 ${ }^{\text {s }}$; Tub-Gal4/+) post-infection, respectively. Meanwhile, the corre- 
sponding flies carrying Drs-GFP reporter also suggested a decrease in the level of Drs in live flies upon infection (95\%, 93\%, 83\% and 45\%) (Figure 2E-G). In addition, the survival situation of individual miRNA high-expressing flies also were observed and recorded upon E. faecalis infection. Compared with the control groups, their survival ability was significantly reduced (Figure 3A-D). Taken together, these results indicate that each miRNA member from miR-959-962 cluster could inhibit the expression of AMP Drs and weaken the resistance to pathogen, to negatively fine tune Drosophila Toll signaling.
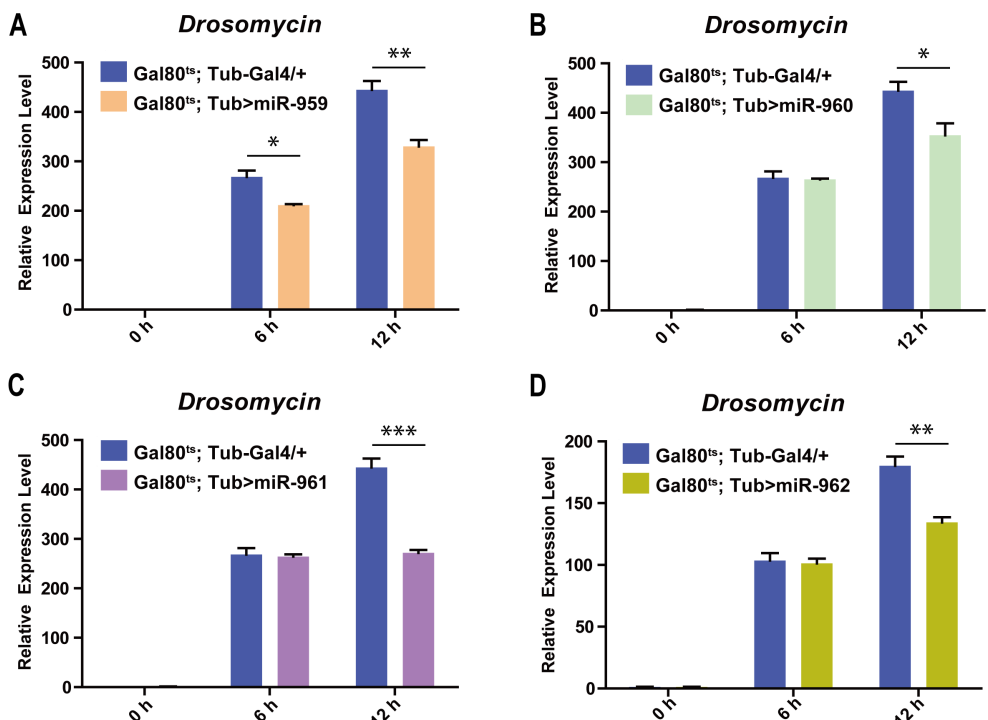

D
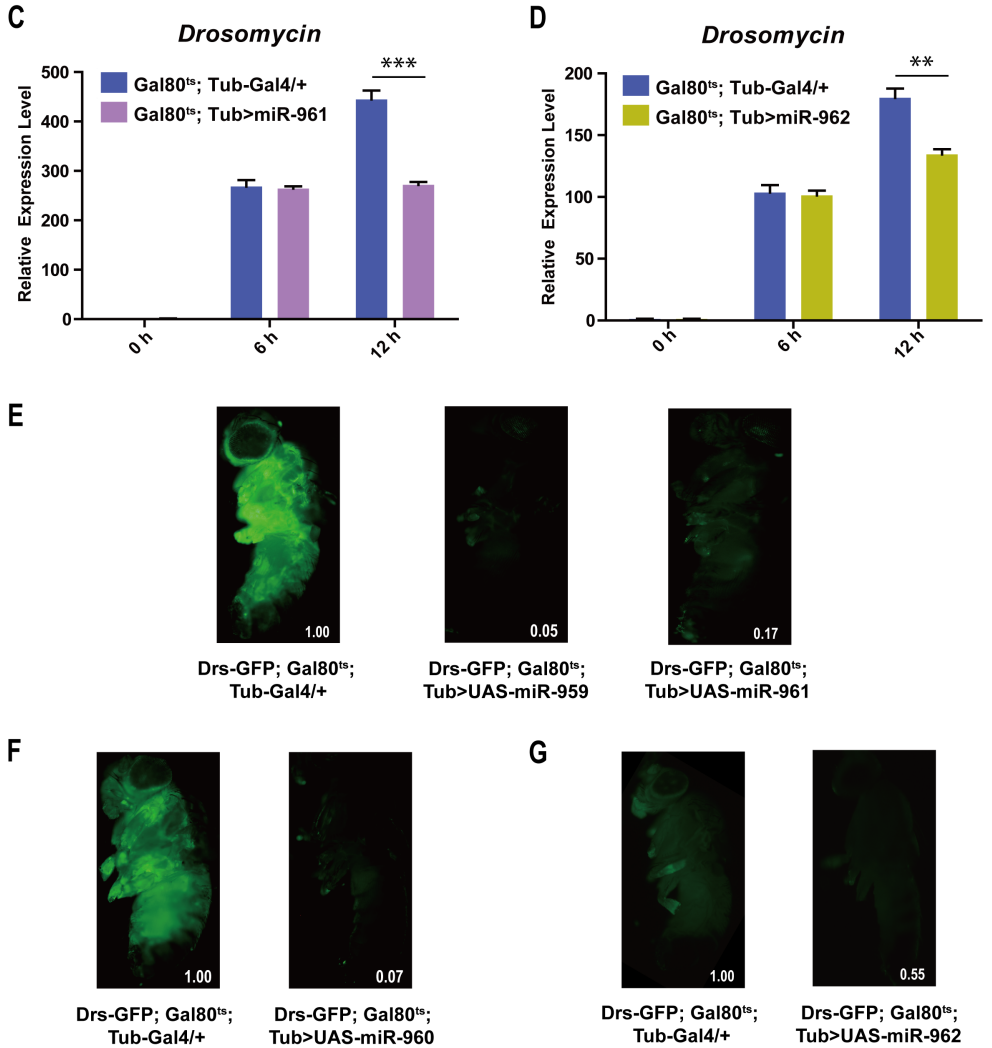

G
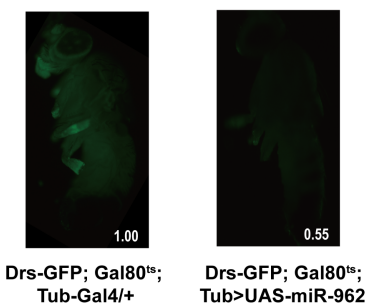

Figure 2. Each of miRNA member from miR-959-962 cluster inhibits the expression of Drs in Drosophila Toll immune response. The expression levels of AMP Drs were examined in miR-959 high-expressing flies (Gal80 $0^{t s}$ Tub > UAS-miR-959) (A), miR-960 high-expressing flies (Gal80 ${ }^{t s}$; Tub > UAS-miR-960) (B), miR-961 high-expressing flies (Gal80 $0^{t s}$; Tub $>$ UASmiR-961) (C), and miR-962 high-expressing flies (Gal80 ${ }^{t s}$; Tub > UAS-miR-962) (D), at 0, 6, and $12 \mathrm{~h}$ upon M. luteus infection. The green-fluorescent in miR-959 high-expressing flies (Drs-GFP; Gal80 ${ }^{\text {ts }}$ Tub > UAS-miR-959, middle) (E), miR960 high-expressing flies (Drs-GFP;Gal80 ${ }^{t s}$; Tub > UAS-miR-960, right) (F), miR-961 high-expressing flies (Drs-GFP;Gal80 $0^{t s}$; Tub > UAS-miR-961, right) (E) and miR-962 high-expressing flies (Drs-GFP;Gal80 ${ }^{\text {ts }}$; Tub > UAS-miR-962, right) (G), and the controls (Drs-GFP; Gal80 ${ }^{\text {ts }}$; Tub-Gal4/+, left) (E-G) carrying with Drs-GFP reporter gene were observed under fluorescent microscope at $12 \mathrm{~h}$ upon $M$. luteus infection. The levels of GFP were quantified using Image J software with the default parameters and their relative level values were marked in the bottom right corner of the image. ${ }^{*} p<0.05 ;{ }^{* *} p<0.01$; *** $p<0.001)$. 
A

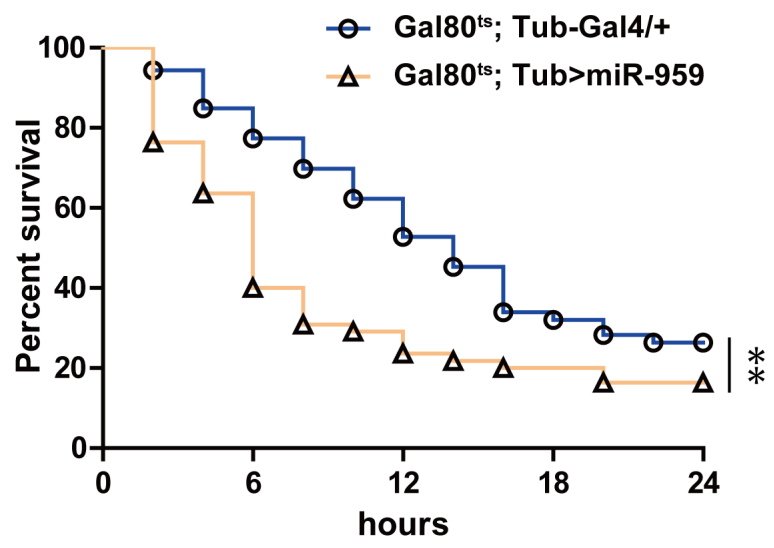

C

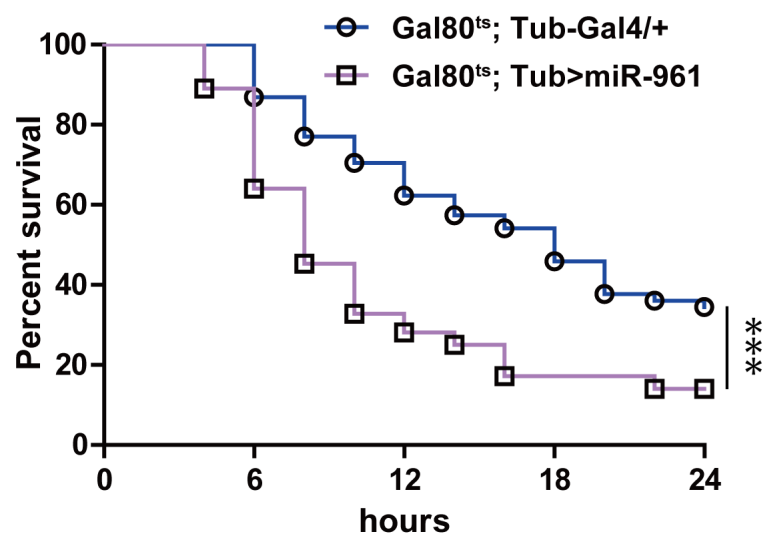

B

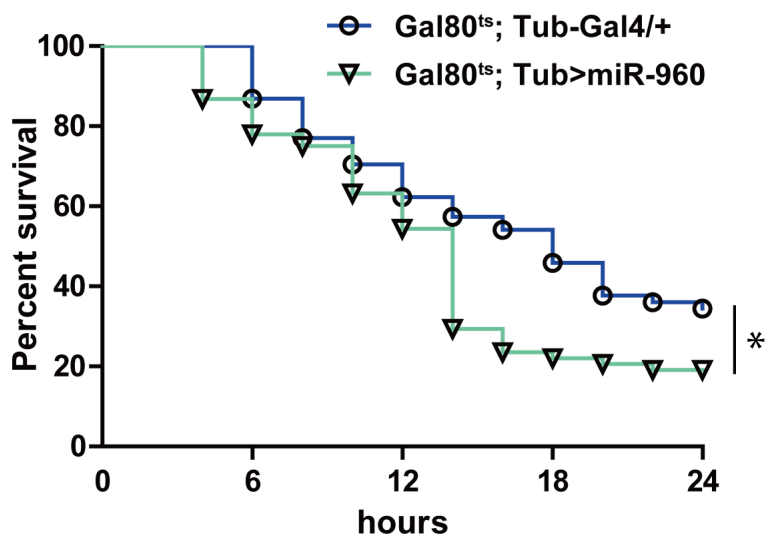

D

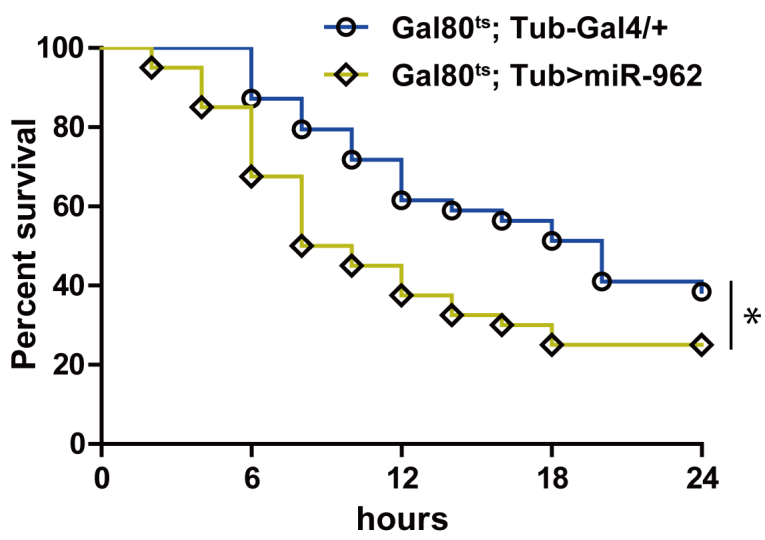

Figure 3. Each of miRNA member from miR-959-962 clusters influences the survival of Drosophila. The changes of the survival rate were observed in miR-959 high-expressing flies (A), miR-960 high-expressing flies (B), miR-961 high-expressing flies (C) and miR-962 high-expressing flies (D), as well as the control flies upon E. faecalis infection. $\left({ }^{*} p<0.05 ;{ }^{* *} p<0.01\right.$; $* * * \quad p<0.001)$.

2.3. The Immune-Related Genes Are Potentially Targeted by miRNA Members from miR-959-962 Cluster In Vitro

In order to further determine how miR-959/960/961/962 regulates the Toll pathway, two algorithms, TargetScan and miRanda, were used to predict the potential target genes of miR-959, miR-960, miR-961, or miR-962. As described in the method, the intersection of two algorithms was acquired as the potential targets. Our results showed that miR959 and miR-960 could bind with the $3^{\prime}$ UTR of tube mRNA, which is the crucial and indispensable effector molecule in the Toll pathway. Moreover, miR-961 and miR-962 had the base complementary pairs with the $3^{\prime}$ UTR of $d l$ mRNA, a key transcription factor that activate the transcription of $A M P$ genes. In addition, miR-962 also had a binding with the 3' UTR of Toll mRNA, a transmembrane factor which transduce signals from extracellular to intracellular. The specific base complementary binding sites are shown in Figure 4A-C. These results suggest that miRNA members from miR-959-962 cluster may play fine-tuning functions at different levels of Toll signals transduction. 


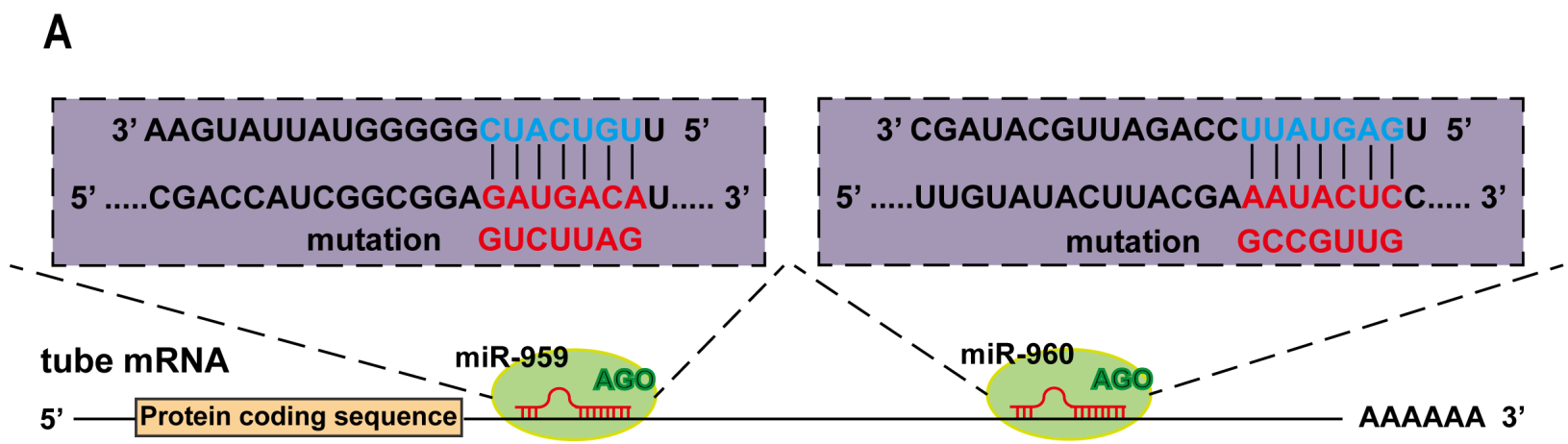

B

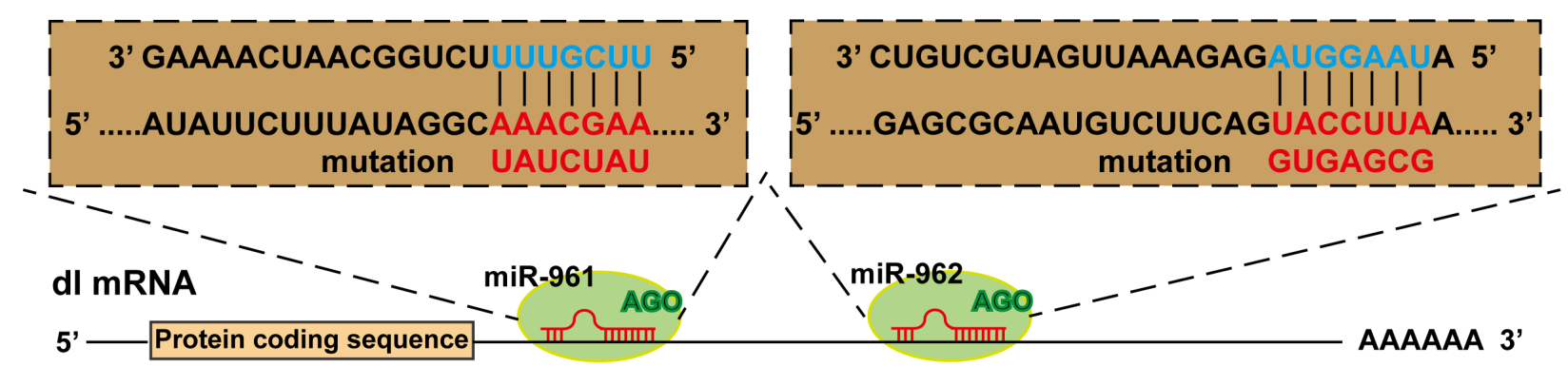

C

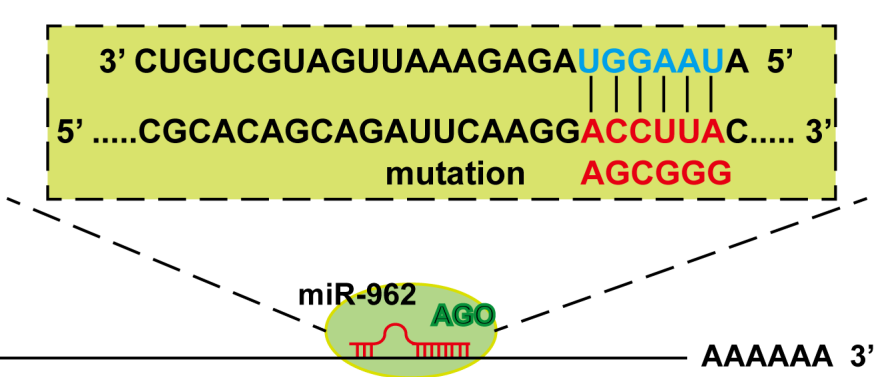

Figure 4. The target genes of four miRNA members from miR-959-962 clusters were predicted. The potential binding sites of miR-959, miR-960, miR-961, and miR-962 in the 3' UTR of tube (A), $d l$ (B) and Toll (C) were present, respectively. The point mutations (red) at the $3^{\prime}$ UTR target sites base pairing to the seed sequence of corresponding miRNA (blue) were performed.

To evaluate the direct targeting relationship between miRNAs and targets, the $3^{\prime}$ UTR sequence of targets ( $t u b e, d l$, and Toll) was respectively recombined to the downstream of the luciferase encoding sequence in the pAc 5.1 insect expression vector, as shown in the Figure 5A,D,G, and the Dual Luciferase Reporter Assay was carried out in Drosophila S2 Cell. The results showed that, compared with the pAc5.1 empty vector, both miR-959 and miR-960 could significantly reduce the activity of the luciferase reporter containing the $3^{\prime}$ UTR of tube (Figure 5B,C). The expression of luciferase reporter carrying with the $3^{\prime}$ UTR of $d l$ could be markedly inhibited both miR-961 and miR-962 (Figure 5E,F). In addition, miR-962 could lower the luciferase activity of Toll 3' UTR report plasmid (Figure 5H). 
A

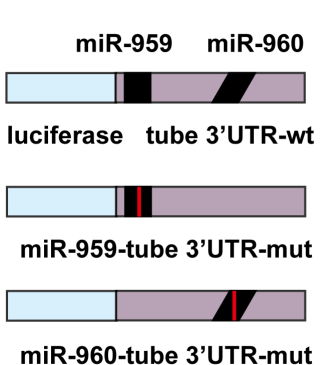

miR-960-tube 3'UTR-mut

D

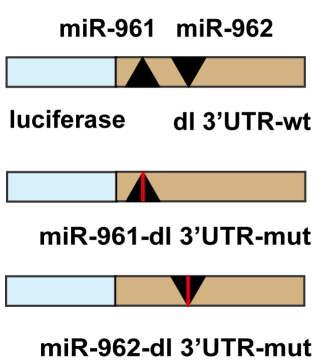

G

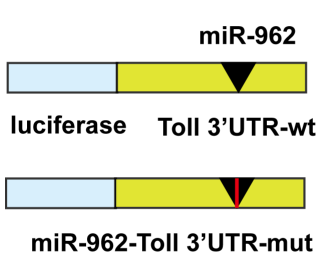

B

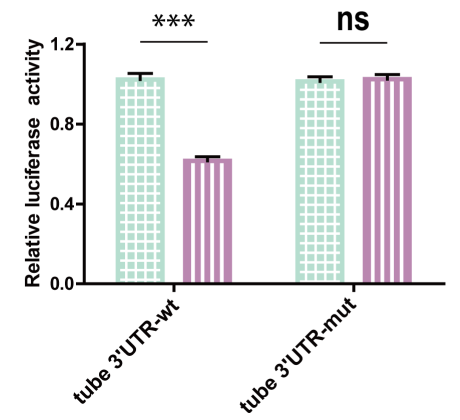

E

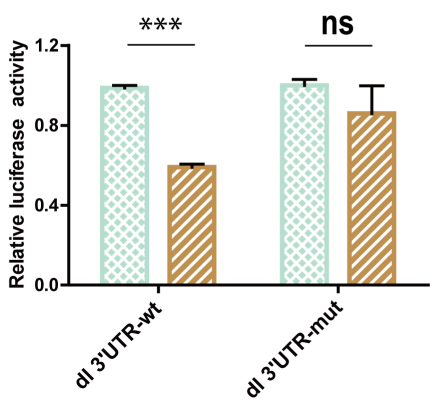

H

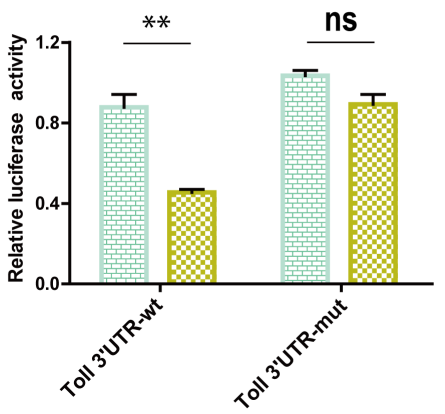

pAc-empty
pAc-miR-962

ns

\section{C}

1+ pAc-empty

pAc-miR-960

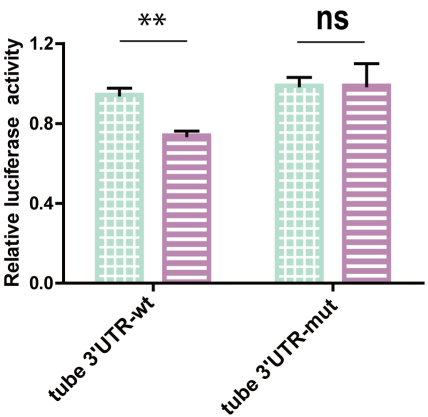

F

pAc-empty

II) pAc-miR-962

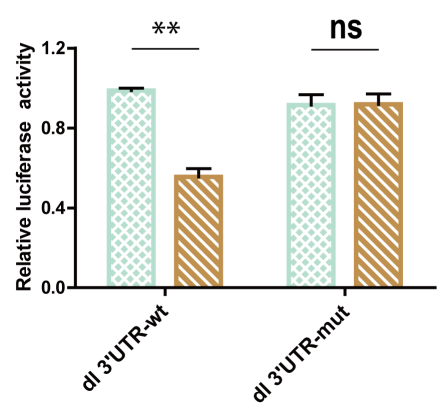

ns

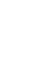


Taken together, our in-vitro results suggest that miR-962 could directly target the $3^{\prime}$ UTR of Toll, miR-959, and miR-960 target the $3^{\prime}$ UTR of tube, and miR-961 and miR-962 target the $3^{\prime}$ UTR of $d l$, indicating that different miRNA members from miR-959-962 cluster function on immunity by targeting different or identical immune-related genes.

\subsection{The miR-959-962 Member Simultaneously or Serperately Target Key Components of Toll Pathway (Tube, dl, and Toll) In Vivo}

To further confirm the reliability of predicted targets of miR-959, miR-960, miR-961 or miR-962 in Drosophila, we performed RT-qPCR analysis in vivo. Our results found that, compared with the control flies, the expression levels of tube mRNA in both miR-959 and miR-960 high-expressing flies were significantly down-regulated upon M. luteus infection (Figure 6A,B); Meanwhile the expression of $d l$ mRNA in both miR-961 and miR-962 highexpressing flies also had a lower level than the controls (Figure 6C,D); In addition, the Toll mRNA level in miR-962 high-expressing flies was a significant reduction (Figure 6E). These suggest the negative correlations between these four miRNAs and corresponding targets in Drosophila.

A

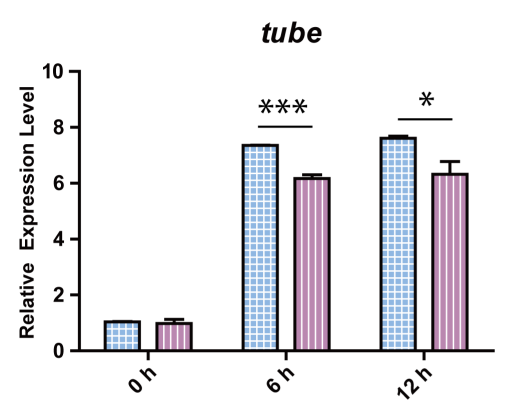

C

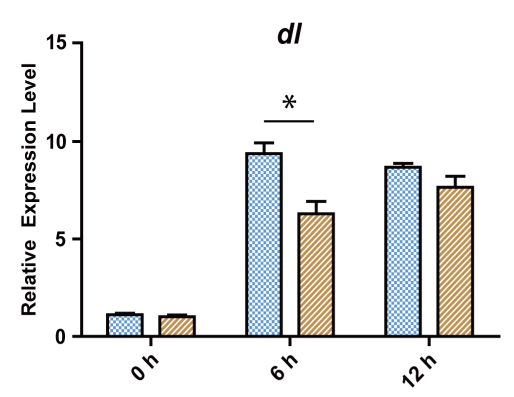

B
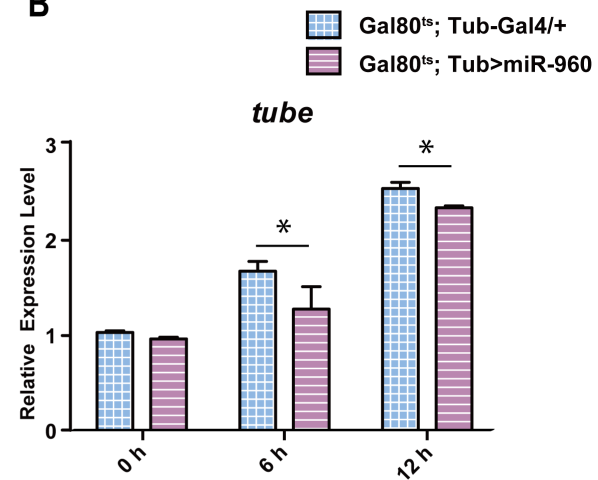

D

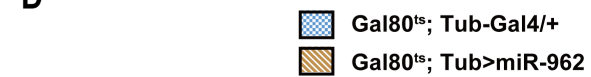

Figure 6. Cont.

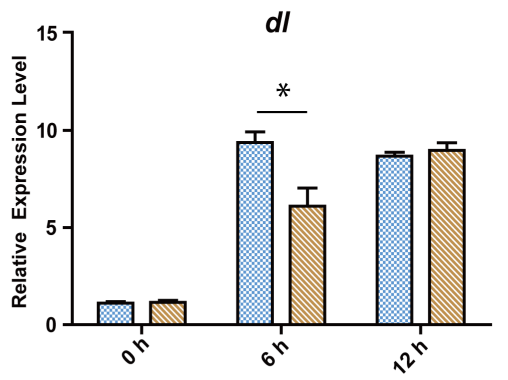




\section{E}

Gal80's; Tub-Gal4/+

Gal80's; Tub>miR-962

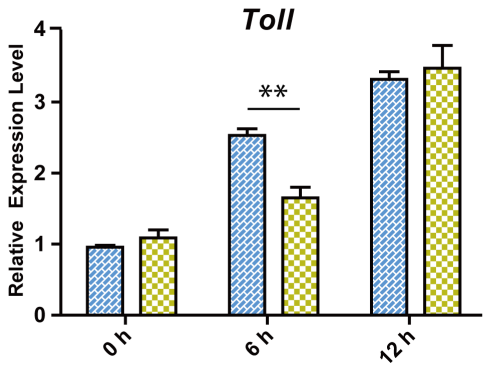

Figure 6. Four miRNA members from miR-959-962 cluster inhibit the expression of its target genes in vivo. The expression levels of tube were respectively tested in miR-959 high-expressing (A) and miR-960 high-expressing flies (B). (C,D) The expression levels of $d l$ in miR-961 high-expressing and miR-962 high-expressing flies were respectively tested. (E) The expression level of Toll in miR-962 high-expressing flies was detected. $\left({ }^{*} p<0.05 ;{ }^{* *} p<0.01 ;{ }^{* * *} p<0.001\right)$.

\subsection{Dynamic Expression Patterns of miR-959-962 Cluster Members in Wild-Type Flies after} M. luteus or PBS Infection

To further explore the important role of this miR-959-962 cluster during Toll pathway response, we monitored the dynamic expression patterns of Drs, miR-959, miR-960, miR961, and miR-962 in wild-type flies with M. luteus infection or PBS (control). Our results found that the levels of Drs in the M. luteus infected flies were significantly higher than the PBS-treated groups at 3, 6, 12, 24, $48 \mathrm{~h}$, and peaked at $24 \mathrm{~h}$ after infection (Figure 7A). Subsequently, we detected the expression levels of miR-959-962 cluster members, respectively. We found that miR-959, miR-960, miR-961, and miR-962 (Figure 7B-E) were respectively significantly increased in the late stage of M. luteus infection. Taken together, we propose that the miR-959-962 cluster could play a crucial role in restoring Drosophila Toll immune homeostasis.

A

Drosomycin

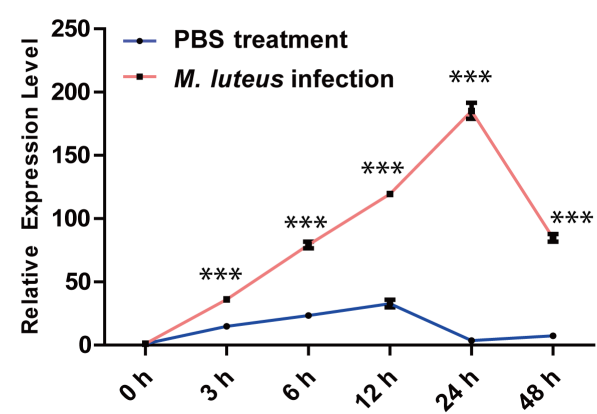

Figure 7. Cont. 
B

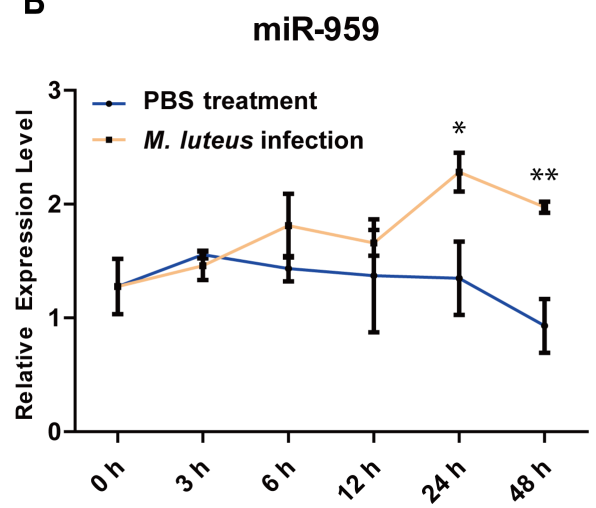

D

miR-961

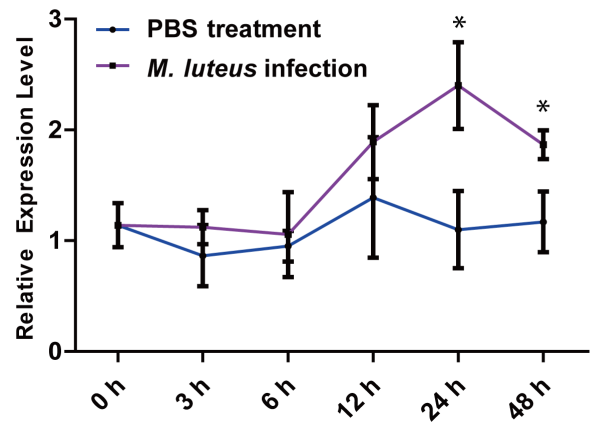

C

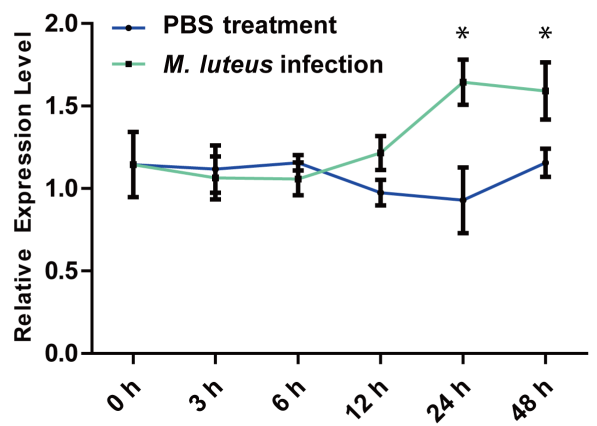

$E$

miR-962

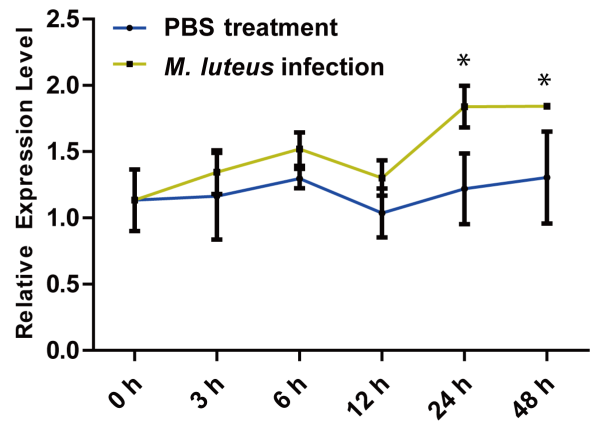

Figure 7. The temporal expression patterns of four miRNAs in the wild-type flies prior to and following M. luteus infection. The dynamic expression changes of Drs (A), miR-959 (B), miR-960 (C), miR-961 (D), miR-962 (E) at six time-points $(0,3,6,12,24$, and $48 \mathrm{~h})$ prior to and following M. luteus or PBS infection, respectively. $\left({ }^{*} p<0.05\right.$; ${ }^{* *} p<0.01$; ${ }^{* * *} p<0.001$; and ns, no significance vs. the control).

\section{Discussion}

Both the deficiency and overactivation of immune response are detrimental to Drosophila. Therefore, the persistence and intensity of the immune response needs to be strictly controlled to maintain the immune homeostasis [39]. At present, increasing evidences have demonstrated that some regulators, such as miRNAs, are involved in negatively regulating the immune signaling to prevent the over-activation of the immune response [34,40,41]. Recently, our group has performed a genome-wide miRNA screening to identify miRNAs regulating Drosophila Toll-mediated innate immune response, employing small-RNA seq and transgenic UAS-miRNA library [37]. Several potential miRNAs have been screened out, followed by in-depth exploration of their regulatory mechanism [35-37]. The current study found that the high-expression of miR-959-962 cluster in flies suppressed antibacterial defenses, evidenced by lower survival rate and a significant decrease of Drs expression in the presence of Gram-positive bacterial challenge.

Despite some reports on the contribution of single miRNA to Drosophila innate immune response have emerged, there are limited reports on how cluster of miRNAs work together. In this study, we demonstrated that each miRNA member of miR-959-962 cluster contributed to the suppression of antibacterial defense by targeting different components of Toll signaling pathway in a combinatory or separate manner, such as miR-959/miR-960 targeting tube, miR-961 repressing $d l$, and miR-962 targeting both $d l$ and Toll. miRNA, perfectly complementary pairing with its target genes (Figure 4), leads to the cleavage and degradation of target mRNA to further block the expression of its protein [42,43]. Our results find that, in the flies with miR-959, miR-960, miR-961, or miR-962 high-expression, 
the corresponding target tube, $d l$, or Toll in mRNA level have a very significant decrease. Therefore, although no data are available, we believe that their protein levels is also sure to be significantly reduced.

MicroRNA clusters widely exist in metazoan genomes, employing with the diversity of their distribution [44]. Most of clustered miRNAs are located in polycistrons and co-expressed with adjacent miRNAs, causing the consistent expression patterns and levels [45,46]. On chromosome 2, the mature miR-959-962 cluster are transcribed from an intron of CG31646 gene and the sequence of miR-963-964 cluster are within neighboring intron in CG31646 gene. A previous study has showed that the six miRNA members from miR-959-964 cluster are probably encoded on a single transcription unit and showed a similar phase and amplitude [47]. Moreover, it has been indicated that the miR-959-964 cluster could inhibit Drosophila immune function against an attenuated strain of Pseudomonas aeruginosa [47]. In our study, of note that miR-960 may execute antibacterial defense only at late $12 \mathrm{~h}$ stage upon infection, while miR-959 may constantly repress the Drs expression at both $6 \mathrm{~h}$ and $12 \mathrm{~h}$ (Figure 2A,B and Figure 3A,B). Meanwhile, miR-961 may contribute more than miR-962 to repress antibacterial defense (Figure 2C,D; Figure 3C,D and Figure 5E,F). Therefore, we speculate that these miRNA of same cluster generated from the same transcripts with similar spatial-temporal expression pattern might have varied stability of half-lives, thus play a synergistic regulatory function on the Toll innate immunity via fine-tuning the different layers of Toll signal transduction (Figure 8).

Remarkably, in our work, the Drs expression and survival analysis shown in Figure 1 were performed under the background of miR-959-962 cluster high-expression or knockout flies (i.e., non-normal physiological conditions), whereas these dynamic expression patterns of four miRNA members of miR-959-962 cluster shown in this Figure 7 were performed under M. luteus infection and PBS treatment in the wild-type flies (i.e., normal physiological conditions). After the high expression of miR-959-962 cluster, Drs expression was downregulated and the survival rate was reduced, implying that the miR-959-962 cluster played a negative regulator role in the Toll pathway (Figure 1). Thus we suggested that under the background of the high-expression of miR-959-962 cluster, miR-959/960/961/962 could inhibit the expression of immune-related target genes (e.g., Toll, tube, and $d l$ ) from the beginning of $M$. luteus infection, and lead to constant suppression of immune response in Drosophila. Therefore, compared with the control group, the flies with miR-959-962 cluster high-expression have an inadequate immune response, and its survival rate has been significantly reduced. Moreover, we analyzed the dynamic expression patterns of four miRNA members of the miR-959-962 cluster in wild-type flies to explore the endogenous role of miR-959/960/961/962 under normal physiological conditions, and we found that compared with PBS treatment groups, all four miRNA members were significantly increased in the late stage of $M$. luteus infection (Figure 7). Taken together, our results suggested that the miR-959-962 cluster plays a negative regulatory role in the later stage of immune response (Figure 8), i.e., in the early stage of M. luteus infection, the expression levels of Drs keep rising, and miR-959/960/961/962 is not up-regulated for avoiding the deficiency of immune response, but in the late stages of infection, in order to avoid the normal tissue damage caused by over-activation of immune response, miR-959/960/961/962 serve as negative regulators to down-regulate Drs expression to help Drosophila to restore to a new immune homeostasis. 


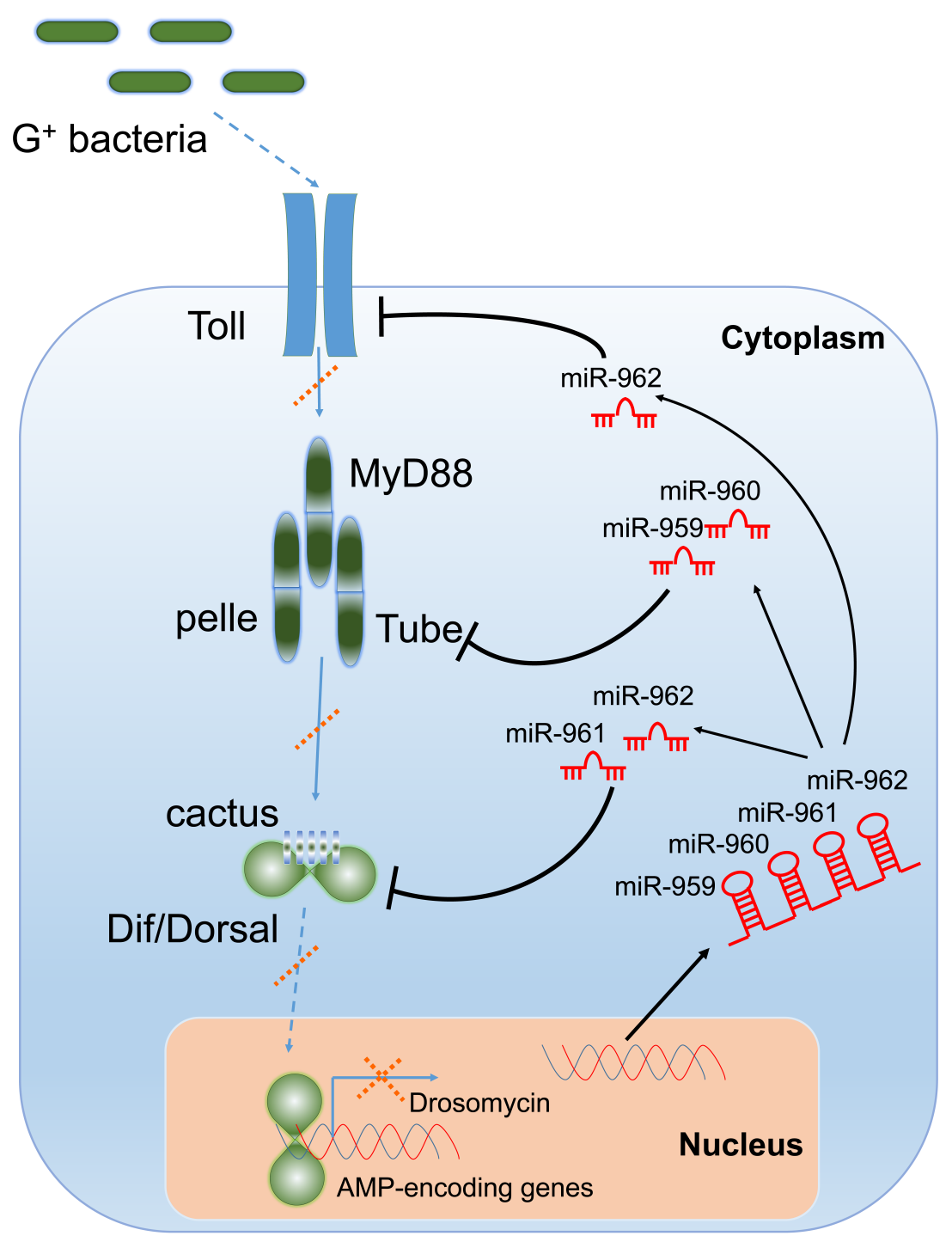

Figure 8. A proposed model. Our results suggested a model in which the miR-959-962 cluster members (red) play a synergistic regulatory function on the Toll innate immunity via fine-tuning the different layers of Toll signal transduction. MiR-959 and miR-960 target the $3^{\prime}$ UTR of tube; miR-961 and miR-962 target the $3^{\prime}$ UTR of $d l$; and miR-962 also target the $3^{\prime}$ UTR of Toll.

In summary, our present studies have revealed the function of miR-959-962 cluster for inhibiting AMP expression and impairing antibacterial defenses. The functions and mechanisms of the four miRNAs from this cluster have also been identified, respectively. Therefore, our results not only identify a new function of miR-959-962 cluster, but also enrich the repertoire of Toll-related immune-modulating miRNA cluster in Drosophila.

\section{Materials and Methods}

\subsection{Drosophila Stocks and Husbandry}

Most flies were obtained from the Bloomington Drosophila Stock Center, including UAS-miR-959/960/961/962 (NO.60615), UAS-miR-959 (NO.60614), UAS-miR-961 (NO.41188), miR-959/960/961/962 KO (NO.58944), except UAS-miR-960 (F001954) and UAS-miR-962 (F001956) from FlyORF. Drosophila was raised on cornmeal-dextrose-yeast agar medium in a light-dark ( $12 \mathrm{~h}$ cycle) incubator at $25{ }^{\circ} \mathrm{C}$ and $60 \%$ humidity. To restrict miRNA overexpression to adulthood with tubulin-Gal $80^{\text {ts }}$, the flies were reared and assayed in either $18^{\circ} \mathrm{C}$ or $29^{\circ} \mathrm{C}$ incubator. 


\subsection{Adult Immune Challenge}

Control and miRNA mutant adult male flies, aged $2-4$ days, were challenged by Micrococcus luteus (M. luteus), a widely used bacterial strain that activates the Toll-mediated immune response to induce the expression of the AMP Drs. Flies were firstly incubated at $29^{\circ} \mathrm{C}$ for $24 \mathrm{~h}$ to activate the overexpression of miRNA. Septic injury was performed by pricking the thorax of the flies with a pulled glass capillary carrying M. luteus suspension mounted on a Nanoject apparatus (WPI, Sarasota, FL, UAS) [48], and then the flies were harvested at specified time points after treatment for RNA extraction and RT-qPCR. For the survival experiment, flies were infected with Gram-positive lethal bacteria, Enterococcus faecalis (E. faecalis), and their survival situation was monitored and recorded for $24 \mathrm{~h}$ post-infection [28].

\subsection{Quantitative RT-PCR Analysis}

Five adult flies per sample group were collected and isolated total RNA with TRIzol Reagent (Invitrogen, Waltham, MA, USA) according to the manufacturer's protocol. RNA concentration and integrity was determined respectively by spectrophotometer and agarose gel separation. cDNA was synthesized using the HiScript ${ }^{\circledR}$ II Q RT SuperMix for qPCR (Vazyme, Nanjing, China). Then quantitative PCR analysis was performed with the StepOnePlus Real-Time PCR System (Applied Biosystems, Foster City, CA, USA) using AceQ ${ }^{\circledR}$ qPCR SYBR Green Master Mix (High ROX Premixed) (Vazyme, Nanjing, China). Each experiment was performed in triplicate and the comparative cycle threshold was used to present a fold change for each specific mRNA/miRNA after normalizing to rp49/U6 snRNA levels. All primers we used in qPCR analyses are listed in Table S1.

\section{4. miRNA Targets Prediction}

The mature sequences of miR-959/960/961/962 and 3' UTR sequences of all genes in Drosophila were respectively acquired from miRBase and FlyBase database. Prediction analysis were carried out locally through employing TargetScan [49] and miRanda [50,51] software packages, applying their default parameters. To increase confidence and reduce false positive of the acquired miRNA-targets, the predicted results of TargetScan and miRanda were overlapped.

\subsection{Recombinant Plasmids Generation}

pAc5.1/V5-HisA insect expression vector was used for the recombinant plasmids construction. To introduce exogenous miR-959, miR-960, miR-961, and miR-962 in Drosophila S2 cells, the pre-miR-959, pre-miR-960, pre-miR-961, and pre-miR-962 sequence were amplified and cloned into pAc5.1/V5-HisA vector to generate pAc-miR-959, pAc-miR-960, pAc-miR-961, and pAc-miR-962 plasmids, respectively. The luciferase coding sequence was subcloned into pAc5.1/V5-HisA to generate pAc-luc. The $3^{\prime}$ UTR sequence of the tube, $d l$, and Toll transcript was respectively inserted to generate pAc-luc-tube $3^{\prime} \mathrm{UTR}$-wt, pAc-luc-dl 3' UTR-wt and pAc-luc-Toll $3^{\prime}$ UTR-wt report plasmids, which were used to express the firefly luciferase. In addition, we also constructed 5 mutants of the above three report plasmids, respectively named pAc-luc-miR-959-tube $3^{\prime}$ UTR-mut, pAc-luc-miR-960tube 3' UTR-mut, pAc-luc-miR-961-dl 3' UTR-mut, pAc-luc-miR-962-dl 3' UTR-mut, and pAc-luc-miR-962-Toll $3^{\prime}$ UTR-mut. In the mutant plasmids, the original binding sites of corresponding miRNA was replaced with other bases without binding. All primers used are listed in Table S2.

\subsection{Cell Transfection and Luciferase Assays}

Drosophila S2 cells were cultured in Drosophila standard medium (Gibco, Waltham, MA, USA) with $10 \%$ fetal bovine serum (Gibco, Waltham, MA, USA) at $28{ }^{\circ} \mathrm{C}$. Before transfection experiment, cells were firstly seeded in 24-well plates (Corning, NY, USA) and cultured for $12 \mathrm{~h}$. In each well, $385 \mathrm{ng}$ miRNA expression plasmid, $100 \mathrm{ng}$ pAc-luc-target $3^{\prime}$ UTR-wt (or pAc-luc-miRNA-target $3^{\prime}$ UTR-mut), and $15 \mathrm{ng}$ pRL were co-transfected into 
cells by using X-tremeGENE HP DNA Transfection Reagent (Roche, Basel, Switzerland) according to manufacturer's instructions. The Renilla luciferase expressed by pRL was used as an internal reference. Dual luciferase assays were performed $48 \mathrm{~h}$ post-transfection with the Dual-Glo luciferase kit (Promega, Madison, WI, USA).

\subsection{Data Processing and Statistical Analysis}

Results from all experiments are presented as means \pm SEM of the data. Statistical analyses were performed using two-tailed Student's t-test, while statistical significance of survival experiment was calculated using the log-rank test (GraphPad Prism 7.04 software). For all statistical analysis, $p<0.05$ was considered significant. All data significantly different from control values are marked with asterisks, ${ }^{*} p<0.05 ;{ }^{* *} p<0.01$; ${ }^{* * *} p<0.001$; and ns, no significance vs. the control.

Supplementary Materials: The following are available online at https: / www.mdpi.com/1422-0 067/22/2/886/s1. Figure S1. The expression levels of miR-959 (A), miR-960 (B), miR-961 (C), and miR-962 (D) in control flies and miR-959-962 cluster high-expressing fly strains were measured before M. luteus infection. Figure S2. The expression levels of miR-959 (A), miR-960 (B), miR-961 (C), and miR-962 (D) were measured in control flies $\left(w^{1118}\right)$ and miR-959-962 cluster knock-out fly strains before M. luteus infection. Figure S3. The expression levels of miR-959 in miR-959 high-expressing flies (A), miR-960 in miR-960 high-expressing flies (B), miR-961 in miR-961 high-expressing flies (C), and miR-962 in miR-962 high-expressing flies (D) were respectively measured before M. luteus infection. Table S1. Primers used for quantitative RT-PCR. Table S2. Primers used for transgene vector construction.

Author Contributions: Conceptualization, R.L., P.J., and F.M.; methodology, R.L., H.Z., and F.M.; software, X.Y. and H.Z.; validation, R.L. and X.Y.; formal analysis, R.L.; investigation, R.L. and X.Y.; resources, P.J. and F.M.; data curation, R.L. and F.M.; writing-original draft preparation, R.L., P.J., and F.M.; writing-review and editing, R.L., P.J., and F.M.; visualization, R.L., P.J., and F.M.; supervision, P.J. and F.M.; project administration, P.J. and F.M.; funding acquisition, F.M. All authors have read and agreed to the published version of the manuscript.

Funding: This research was funded by the National Natural Science Foundation of China (No. 31970477 and No. 31572324), the Natural Science Foundation from Jiangsu Province (No. BK20191368), and the APC was funded by the Priority Academic Program Development of Jiangsu Higher Education Institutions.

Institutional Review Board Statement: Not applicable.

Informed Consent Statement: Not applicable.

Data Availability Statement: All relevant data are within the manuscript and its Supporting Materials.

Acknowledgments: We truly appreciate the contributions of all authors to this Original Research. We also thank all reviewers and editors who assisted us and provided thorough comments and invaluable suggestions. We are also grateful to the Bloomington Stock Center and FlyORF for providing fly stocks.

Conflicts of Interest: The authors declare no conflict of interest. The funders had no role in the design of the study; in the collection, analyses, or interpretation of data; in the writing of the manuscript, or in the decision to publish the results.
Abbreviations
miRNA microRNA
Drs Drosomycin
dl dorsal
$3^{\prime}$ UTR $\quad 3^{\prime}$ untranslated region
imd immune deficiency
AMP antimicrobial peptide 
$\begin{array}{ll}\text { M. luteus } & \text { Micrococcus luteus } \\ \text { E. faecalis } & \text { Enterococcus faecalis } \\ \text { Drs-GFP } & \text { Drosomycin-green fluorescent protein }\end{array}$

\section{References}

1. Ayyar, K.K.; Reddy, K.V.R. MAPK and NF-kappaB signalling pathways regulate the expression of miRNA, let-7f in human endocervical epithelial cells. J. Cell. Biochem. 2018, 119, 4751-4759. [CrossRef] [PubMed]

2. Parkin, J.; Cohen, B. An overview of the immune system. Lancet 2001, 357, 1777-1789. [CrossRef]

3. Kounatidis, I.; Ligoxygakis, P. Drosophila as a model system to unravel the layers of innate immunity to infection. Open Biol. 2012, 2, 120075. [CrossRef] [PubMed]

4. Hoffmann, J.A. The immune response of Drosophila. Nature 2003, 426, 33-38. [CrossRef] [PubMed]

5. Lemaitre, B.; Hoffmann, J. The host defense of Drosophila melanogaster. Annu. Rev. Immunol. 2007, 25, 697-743. [CrossRef] [PubMed]

6. Hultmark, D. Drosophila immunity: Paths and patterns. Curr. Opin. Immunol. 2003, 15, 12-19. [CrossRef]

7. Valanne, S.; Wang, J.H.; Ramet, M. The Drosophila Toll signaling pathway. J. Immunol. 2011, 186, 649-656. [CrossRef]

8. Michel, T.; Reichhart, J.M.; Hoffmann, J.A.; Royet, J. Drosophila Toll is activated by Gram-positive bacteria through a circulating peptidoglycan recognition protein. Nature 2001, 414, 756-759. [CrossRef]

9. Bischoff, V.; Vignal, C.; Boneca, I.G.; Michel, T.; Hoffmann, J.A.; Royet, J. Function of the drosophila pattern-recognition receptor PGRP-SD in the detection of Gram-positive bacteria. Nat. Immunol. 2004, 5, 1175-1180. [CrossRef]

10. Wang, L.; Weber, A.N.; Atilano, M.L.; Filipe, S.R.; Gay, N.J.; Ligoxygakis, P. Sensing of Gram-positive bacteria in Drosophila: GNBP1 is needed to process and present peptidoglycan to PGRP-SA. EMBO J. 2006, 25, 5005-5014. [CrossRef]

11. Gottar, M.; Gobert, V.; Matskevich, A.A.; Reichhart, J.M.; Wang, C.; Butt, T.M.; Belvin, M.; Hoffmann, J.A.; Ferrandon, D. Dual detection of fungal infections in Drosophila via recognition of glucans and sensing of virulence factors. Cell 2006, 127, 1425-1437. [CrossRef] [PubMed]

12. Weber, A.N.; Delamasure, S.T.; Hoffmann, J.A.; Lelievre, E.; Gascan, H.; Ray, K.P.; Morse, M.A.; Imler, J.L.; Gay, N.J. Binding of the Drosophila cytokine Spatzle to Toll is direct and establishes signaling. Nat. Immunol. 2003, 4, 794-800. [CrossRef] [PubMed]

13. Hu, X.; Yagi, Y.; Tanji, T.; Zhou, S.; Ip, Y.T. Multimerization and interaction of Toll and Spatzle in Drosophila. Proc. Natl. Acad. Sci. USA 2004, 101, 9369-9374. [CrossRef] [PubMed]

14. Morisato, D.; Anderson, K.V. The spatzle gene encodes a component of the extracellular signaling pathway establishing the dorsal-ventral pattern of the Drosophila embryo. Cell 1994, 76, 677-688. [CrossRef]

15. Schneider, D.S.; Jin, Y.; Morisato, D.; Anderson, K.V. A processed form of the Spatzle protein defines dorsal-ventral polarity in the Drosophila embryo. Development 1994, 120, 1243-1250.

16. Delamasure, S.T.; Bilak, H.; Capovilla, M.; Hoffmann, J.A.; Imler, J.L. Drosophila MyD88 is required for the response to fungal and Gram-positive bacterial infections. Nat. Immunol. 2002, 3, 91-97. [CrossRef]

17. Horng, T.; Medzhitov, R. Drosophila MyD88 is an adapter in the Toll signaling pathway. Proc. Natl. Acad. Sci. USA 2001, 98, 12654-12658. [CrossRef]

18. Xiao, T.; Towb, P.; Wasserman, S.A.; Sprang, S.R. Three-dimensional structure of a complex between the death domains of Pelle and Tube. Cell 1999, 99, 545-555. [CrossRef]

19. Ip, Y.T.; Reach, M.; Engstrom, Y.; Kadalayil, L.; Cai, H.; Gonzalez-Crespo, S.; Tatei, K.; Levine, M. Dif, a dorsal-related gene that mediates an immune response in Drosophila. Cell 1993, 75, 753-763. [CrossRef]

20. Lemaitre, B.; Meister, M.; Govind, S.; Georgel, P.; Steward, R.; Reichhart, J.M.; Hoffmann, J.A. Functional analysis and regulation of nuclear import of dorsal during the immune response in Drosophila. EMBO J. 1995, 14, 536-545. [CrossRef]

21. Wu, L.P.; Anderson, K.V. Regulated nuclear import of Rel proteins in the Drosophila immune response. Nature 1998, 392, 93-97. [CrossRef] [PubMed]

22. Lemaitre, B.; Reichhart, J.M.; Hoffmann, J.A. Drosophila host defense: Differential induction of antimicrobial peptide genes after infection by various classes of microorganisms. Proc. Natl. Acad. Sci. USA 1997, 94, 14614-14619. [CrossRef] [PubMed]

23. Hetru, C.; Hoffmann, J.A. NF-kappaB in the immune response of Drosophila. Cold Spring Harb. Perspect. Biol. 2009, 1, a000232. [CrossRef] [PubMed]

24. Myllymaki, H.; Valanne, S.; Ramet, M. The Drosophila imd signaling pathway. J. Immunol. 2014, 192, 3455-3462. [CrossRef] [PubMed]

25. Kambris, Z.; Brun, S.; Jang, I.H.; Nam, H.J.; Romeo, Y.; Takahashi, K.; Lee, W.J.; Ueda, R.; Lemaitre, B. Drosophila immunity: A large-scale in vivo RNAi screen identifies five serine proteases required for Toll activation. Curr. Biol. 2006, 16, 808-813. [CrossRef] [PubMed]

26. Chamy, L.E.; Leclerc, V.; Caldelari, I.; Reichhart, J.M. Sensing of 'danger signals' and pathogen-associated molecular patterns defines binary signaling pathways 'upstream' of Toll. Nat. Immunol. 2008, 9, 1165-1170. [CrossRef]

27. Haghayeghi, A.; Sarac, A.; Czerniecki, S.; Grosshans, J.; Schock, F. Pellino enhances innate immunity in Drosophila. Mech. Dev. 2010, 127, 301-307. [CrossRef] 
28. Valanne, S.; Myllymaki, H.; Kallio, J.; Schmid, M.R.; Kleino, A.; Murumagi, A.; Airaksinen, L.; Kotipelto, T.; Kaustio, M.; Ulvila, J.; et al. Genome-wide RNA interference in Drosophila cells identifies $\mathrm{G}$ protein-coupled receptor kinase 2 as a conserved regulator of NF-kappaB signaling. J. Immunol. 2010, 184, 6188-6198. [CrossRef]

29. Reed, D.E.; Huang, X.M.; Wohlschlegel, J.A.; Levine, M.S.; Senger, K. DEAF-1 regulates immunity gene expression in Drosophila. Proc. Natl. Acad. Sci. USA 2008, 105, 8351-8356. [CrossRef]

30. Gordon, M.D.; Dionne, M.S.; Schneider, D.S.; Nusse, R. WntD is a feedback inhibitor of Dorsal/NF-kappaB in Drosophila development and immunity. Nature 2005, 437, 746-749. [CrossRef]

31. Xiao, C.; Rajewsky, K. MicroRNA control in the immune system: Basic principles. Cell 2009, 136, 26-36. [CrossRef] [PubMed]

32. Stark, A.; Brennecke, J.; Russell, R.B.; Cohen, S.M. Identification of Drosophila MicroRNA targets. PLoS Biol. 2003, 1, e60. [CrossRef] [PubMed]

33. Ha, J.; Kim, H.; Yoon, Y.; Park, S. A method of extracting disease-related microRNAs through the propagation algorithm using the environmental factor based global miRNA network. BioMed Mater. Eng. 2015, 26 (Suppl. 1), S1763-S1772. [CrossRef]

34. Lee, G.J.; Hyun, S. Multiple targets of the microRNA miR-8 contribute to immune homeostasis in Drosophila. Dev. Comp. Immunol. 2014, 45, 245-251. [CrossRef] [PubMed]

35. Li, S.; Li, Y.; Shen, L.; Jin, P.; Chen, L.; Ma, F. miR-958 inhibits Toll signaling and Drosomycin expression via direct targeting of Toll and Dif in Drosophila melanogaster. Am. J. Physiol. Cell Physiol. 2017, 312, C103-C110. [CrossRef]

36. Li, R.; Huang, Y.; Zhang, Q.; Zhou, H.; Jin, P.; Ma, F. The miR-317 functions as a negative regulator of Toll immune response and influences Drosophila survival. Dev. Comp. Immunol. 2019, 95, 19-27. [CrossRef]

37. Li, Y.; Li, S.; Li, R.; Xu, J.; Jin, P.; Chen, L.; Ma, F. Genome-wide miRNA screening reveals miR-310 family members negatively regulate the immune response in Drosophila melanogaster via co-targeting Drosomycin. Dev. Comp. Immunol. 2017, 68, 34-45. [CrossRef]

38. Li, S.; Xu, J.; Sun, L.; Li, R.; Jin, P.; Ma, F. Drosophila miR-964 modulates Toll signaling pathway in response to bacterial infection. Dev. Comp. Immunol. 2017, 77, 252-258. [CrossRef]

39. Li, R.; Zhou, H.; Jia, C.; Jin, P.; Ma, F. Drosophila Myc restores immune homeostasis of Imd pathway via activating miR-277 to inhibit imd/Tab2. PLoS Genet. 2020, 16, e1008989. [CrossRef]

40. Choi, I.K.; Hyun, S. Conserved microRNA miR-8 in fat body regulates innate immune homeostasis in Drosophila. Dev. Comp. Immunol. 2012, 37, 50-54. [CrossRef]

41. Chen, C.Z.; Schaffert, S.; Fragoso, R.; Loh, C. Regulation of immune responses and tolerance: The microRNA perspective. Immunol. Rev. 2013, 253, 112-128. [CrossRef] [PubMed]

42. Sun, W.; Julie Li, Y.S.; Huang, H.D.; Shyy, J.Y.; Chien, S. microRNA: A master regulator of cellular processes for bioengineering systems. Annu. Rev. Biomed. Eng. 2010, 12, 1-27. [CrossRef] [PubMed]

43. Bartel, D.P. MicroRNAs: Genomics, biogenesis, mechanism, and function. Cell 2004, 116, 281-297. [CrossRef]

44. Zhang, Y.; Zhang, R.; Su, B. Diversity and evolution of MicroRNA gene clusters. Sci. China C Life Sci. 2009, 52, 261-266. [CrossRef]

45. Cullen, B.R. Transcription and processing of human microRNA precursors. Mol. Cell 2004, 16, 861-865. [CrossRef]

46. Baskerville, S.; Bartel, D.P. Microarray profiling of microRNAs reveals frequent coexpression with neighboring miRNAs and host genes. RNA 2005, 11, 241-247. [CrossRef]

47. Vodala, S.; Pescatore, S.; Rodriguez, J.; Buescher, M.; Chen, Y.W.; Weng, R.; Cohen, S.M.; Rosbash, M. The oscillating miRNA 959-964 cluster impacts Drosophila feeding time and other circadian outputs. Cell Metab. 2012, 16, 601-612. [CrossRef]

48. Neyen, C.; Bretscher, A.J.; Binggeli, O.; Lemaitre, B. Methods to study Drosophila immunity. Methods 2014, 68, 116-128. [CrossRef]

49. Lewis, B.P.; Burge, C.B.; Bartel, D.P. Conserved seed pairing, often flanked by adenosines, indicates that thousands of human genes are microRNA targets. Cell 2005, 120, 15-20. [CrossRef]

50. Betel, D.; Wilson, M.; Gabow, A.; Marks, D.S.; Sander, C. The microRNA.org resource: Targets and expression. Nucleic Acids Res. 2008, 36, D149-D153. [CrossRef]

51. Enright, A.J.; John, B.; Gaul, U.; Tuschl, T.; Sander, C.; Marks, D.S. MicroRNA targets in Drosophila. Genome Biol. 2003,5 , R1. [CrossRef] [PubMed] 